Fon Organen durch Mehrgebrauch und Ubung. In Wahrheit ist naturlich mit dieser Gleichsetzung noch keine Erklärung gegeben, denn der Mechanismus der U̇bungshypertrophie bedarf selbst noch der Erklärung, Daß aber die Dinge noch tiefer. liegen, verraten eigentümliche Erfahrungen, die man bei Versuchen gemacht hat, bei normalen Organismen durch Einpflanzung eines Organes oder Organstückes einen Mehrbesitz zu erzielen. Dies gelingt nicht; das Eingepflanzte geht innerhalb kurzer Zeit zugrunde. Solche Versuche wurden angestellt, um als Gegenstück zu Krankheitsbildern, die auf dem Fehlen gewisser Organe mit innerer Sekretion beruhen, solche $z u$ erzeugen, von denen man annimmt, daB sie durch eine erhebliche Steigerung der normalen Leistung des betreffenden Organes zustande kommen. Ganz anders wird die Sache, wenn man vorher das der Untersuchung dienende Organ entfernt hat und nun ron einem anderen Individuum der gleichen Art das fehlende Organ ersetzt. Jetzt gelingt die Implantation, erkennbar daran, daB sowohl der morphologische wie auch der funktionelle Bewreis für das Erhaltenbleiben des implantierten Organes erbracht werden kann. Der amerikanische Chirurg Halsted hat diesen Tatbestand durch den Satz zu formulieren versucht, daß erst der Ausfall die Bedingungen zur Ermöglichung des anatomischen und funktionellen Implantationserfolges schafft. Hier tritt demnach ein neuer Faktor, der Ausfall mit seinen Folgen, in den Mechanismus für die Entstehung der nachfolgenden Anpassung ein. Der Ausfall mit seinen Folgen $\mathrm{muB}$ in doppelter Weise wirken, indem er einerseits den Boden verändert, auf dem Einwachsen stattfinden soll, andererseits die individuellen Fremdzellen wachstumsund assimilationsfähig macht, was sie vorher wicht waren. Woriu die Bodenveränderungen bestehen könnten, darüber besitzen wir noch keinen Einblick, wohl aber können wir eine Aussage darüber machen, was Zellen zum Wachstum befähigt macht. Die gröBte Wachstumsbefähigung besitzen Zellen im Embryonalzustande. Es liegt daher nahe, die Annahme zu machen, dab in die Mechanik derjenigen Anpassungsvorgänge, zu deren Ermöglichung gesteigertes Zellwachstum gehört, die Unwandlung der Zellen in solche mit Embryonalcharakter gehört. Möglicherweise spielt dieser Umwandlungsproze $B$ auch dort eine maßgebende Rolle, wo es sich um den Vorgang der Leistungssteigerung nicht völlig fehlender, sondern nur in ithrer Menge verminderter Organteile handelt. Die Gesamtheit dieser Ausführungen sollte zeigen, daß wir jetzt zwei Faktoren kennen, die in die Mechanik der angenblicklich geschilderten Anpassungsvorgänge eingehen, nämlich Ausfall und Umwandlung von Zellen $z u$ solchen mit embryonalen Eigenschaften.

Nur andeutungsweise sei hier darauf hingewiesen, daß nach $E$ hrlichs geistroller Seitenkettentheorie der ,Ausfall" ein sehr wichtiges Glied in der Kette der Ereignisse ist, die zur Immunisierung bei dex Injektion von Heilsera führen, Immunisierungen, die wir nicht anders als zu den Anpassungsvorgängen rechnen können. Es mub auf manche bekannte vorzitgliche Darstellungen der Ehrlichschen Lehre in der Literatur dex Immunitätsforschung verwiesen werden.

Am Schlul unserer Ausführungen angelangt, sollen die wesentliohsten Gesichtspunkte noch ein. mal hervorgehoben werden, welehe aus einer Betrachtung dex expeximentell beobachtbaren. Tatsachen der funktionellen Anpassung sich daxbieten. Vielleicht am wichtigsten erscheint mir die Erkenntnis, daß das Vermögen, welches in den Anpassungsvorgängen zutage tritt, eine elementare Eigenschaft jeder belebten Substanz ist, ihr in gleicher Weise und unauslösbar innewohnend, wie andere elementare Lebenseigenschaften, ohne welche wix uns den Bestand des Lebens nicht denken können. Mit dieser Auffassung steht im Einklang, daB Anpassung beobachtet werden kann bis herab zu den letzten Einheiten, welche der Experimentator noch im Besitz des Vollzugs der physiologischen Funktionen zu isolieren vermag. Ferner stimmt damit überein, daß die Anpassung nicht notwendiger weise der Zeit bedarf, um in Wirksamkeit zu treten, sondern daß sie bereit steht, sobald die Erfordernisse es erheischen. Die Anpassung kann auch völig unabhängig von dem morphologischen Aufbau zur Entfaltung kommen, eine Unabhängigkeit, die eigentlich ein Postulat ist, wenn man sich vergegenwärtigt, daß die Form zelbst ein Geschaffenes und nicht ein Schöpfer der Funktion ist, was freilich nicht mit den sekundären funktionellen Leistungen gewisser Formen rerwechselt werden dar. Viele Biologen sind geneigt, dem Zufall einen nicht geringen Anteil an dem Werden der Organismen beizumesseu. Die hier vorgetragene Auffassung von dem Anpassungsvermögen als einex elementaren Eigenschaft der belebten Substanz entkleidet dieselbe des gedachten Charakters der Zufälligkeit. Sie sind Regeln unterworfen, die zwar anderer Art, aber nicht minder streng sind als diejenigen für die Vorgänge in der uabelebten Natur. Es steht zu hoffen, daB dic Behandlung der Anpassung als eines Problems der, allgemeinen, funktionel gerichteten Physiologie berufen sein wird, an der Lösung von Fragen mitzuwirken, die mehr erstreben als einen Einblick in die Geschehnisse, welche die individuelle Existenz ausmachen.

\section{Vom mechanischen Äther zur elektrischen Materie}

Von Prof: Dr. M. Barn, Berlin.

Es ist noch nicht lange her, da wat die physikalische Literatur mit Abhandlungen erfüllt, die die mechanischen Eigenschaften des Athers 
rörterten, und einige Ubberreste solcher Betrachtungen haben sich bis auf unsere Tage erhalten. Im ganzen aber hat sich die Physik nenen Anschauungen zugewandt, die man mit einem kurzen Schlagwort als die Lehre von der elehtrischen Natur der Materie bezeichnen könnte.

Es handelt sich dabei un eine grindliche Umstellung der Gedankonwelt; was früher als das Einfache, Primäre galt (die Mechanik), wird heute zum Abgeleiteten. Sekundären, und nm gekehrt wird das, was ehemals der Zurückführung auf elementare Gegebenheiten bedürftig schien (der Ather als Träger dex elektromagnetischen Erscheinungen), zum Fundament des ganzen. Gebäudes der Wissenschaft gemacht. Es lohnt der Míhe, diesen Wandel der Ideen zu ïberblicken und sich klar zu machen, ob er einen Fortsehritt der Erkenntnis bedeutet.

Die ,Physik des Athers" nahm ihren Ausgang am Anfange des 19. Jahrhunderts mit der Optik. In dieser Zeit hatte die Mechanik jene Vollkommenheit erreicht, die sie zur Königin der Wissenschaften machte. Neben der Mechanik der Massenpunkte, die ihre Triumphe in der Astronomie feierte, war die Mechanik der kontinuierlich verbreiteten Medien, die elastische Theorie der Gase, Flüssigkeiten und festen Körper, entwickelt worden. So konnte es nicht ausbleiben, das die mechanischen Gesetze auch auf die optischen Vorgänge angewandt wurden. Die Lichtwellen wurden mit den elastischen Wellen in materiellen Körpern verqhichea; es entstand die erste elastische Theorie des Athers.

In elastischen Kömern gibt es aber zwei Arten von Wellen, solche, bei denen die Teilchen in der Richtung der Wellenfortschreisung hin- und herschwingen (longitudinale Wellen), und solche, bei denen die Téilchen quer zux Fortpflanzungsrichtung pendeln (traasversale Wellen). Es ließ sich nun nachweisen, daß die Lichtwellen streng transversal sind; bis zum heutigen Tage hat man keine Andeutung optischer Longitudinatwellen gefunden. Da nun longitudinale Schwingungen offenbar mit Dichteändevungen verknüpft sind, so muBte man aus dem Fehlen der longitudinalen Lichtwellen schließen, daB der Ather sich nicht (wie ein Gas) zusammendricken läBt; andererseits aber muB er äuBerst elastisch sein, da er (wie ein fester Körper) transversale Wellen sehr gut fortpflanzt.

Man kam also zu dem SchluB: Der Ather ist ein inkompressibler, vollkommen elastischer, fester Körper. Und trotzdem bewegen sich durch den Ather die Himmelskörper mit ungeheuren Gesehwindigkeiten völlig reibungs- und störungsfrei.

Neben dieser Denkschwierigkeit tràten bei der Durchführung der elastischen Lichttheorie noch andere Mängel zutage, so dab es keines allzu starken Stoßes bedurfte, um diese Lehre zu stürzen.
Diesur StoB kam durch die elektromagnetische Lichtheorie von Maxuell. Danach wird der Ather nicht mehr als elastischer Körper vorgestellt, aber er behält doch die Natur eines kontinuierlich verbreiteten Mediums mit bestimmten Eigenschaften, die als elektrisches und magnetisehes Feld in die Erscheinung treten. Schnelle Schwinguugen der Felder sind Lichtwellen, ihre Geschwindigkeit läbt sich durch rein elektromagnetische Messungen ermitteln.

Diese Theorie erfordert eine beträchtliche $\mathrm{Ab}$ straktion. Was ist eigentlich dieser Ather, der zwar ein kontinuierliches Medium, aber von den bekannten Körpern, seien sie gasförmig, flüssig oder fest. verschieden sein soll? Das Bedürfnis nach Vorstellbarkeit, Bildhaftigkeit der Theorien fordert Zurückführung auf Bekanntes, und schon Maxwell ist diesem Bedürfnis durch Angabe mechanischer Modelle füx gewisse Eigenschaften des Athers entgegengekommen. Später ist die Frage der Zurückführung des Maxwellschen Athers auf mechanische Grundlagen eine ganze Wissenschaft geworden. Besonders groBe Erfolge hat Bjertenes erzielt, der den Äther als Flüssigkeit und die elektrischen Ladungen als pulsierende Kugeln darin auffaßte, eine Lehre, die von Korn weiter ausgebant wurde und noeh heute vertreten wird.

Neue Entdeckungen sind auf diesen Wegen nicht gemacht worden. Gleichwohl wäre das Interesse daran vielleicht erhalten geblieben, hätte nicht die Relativitätstheorie die Vorstellung eines materiellen Äthers überhaupt unmöglich gemacht. Allerdings erfordert sie eine noch hohere $\mathrm{Ab}$ straktion, einen stärkeren Verzicht auf die gewöhnliche Anschaulichkeit, und darum blickt mancher Physiker wehmütig zurück auf die gute alte Zeit des soliden Athers (ohne es offentlich einzugestehen, wie der Amerikaner auf die, good old colonial times").

Die Enkenntnis schreitet aach der Tiefe durch Abstraktionen fort, in die Breite aber durch die lebendige Anschauung, und wenn die Theorie nichts anderes zuwege gebracht hätte, als die prinzipielle Undurchführbarkeit aller mechanischen Bilder des Äthers und seiner Eigenschaften zu erweisen, so hätte sie der Forschung einen schlechten Dienst geleistet. Aber ein Rückblick auf die Physik in den letzten Jahren zeigt deutlich, daß die Experimentierkunst in enger Fühlung mit der Theorie ihre größten Erfolge erzielt hat; also muB diese wohl der Anschauung, der Intuition neue, lebendige Bilder liefern, die fruchtbarer wirken als die Mechanisierung des Athers. Das ,elektromagnetische Weltbild" der heutigen Physik mag in den Grundlagen abstrakt genannt werden; in seinem Ausbau ist es reich an Farben und Formen.

Die Quelle dieses Reichtums beruht auf der Vereinigung der abstrakten Maxwellschen Athervorstellung (bzw. threr relativistischen Vertiefung) mit der Atomistik. 
Nicht nur die Materie wird heute atomistisch a ufgefaBt, die Elektrizität selber wird in Atome, Elektronen genannt, aufgelöst. Atome und Elektronen aber sind anschaulich leicht zu fassen; auch wer dem Formelwust der Maxwellschen Elektrizitätstheorie mißtrauisch gegenübersteht, kann mit den Bewegungen und wechselseitigen Wirkungen der Elektronen und Atome erfolgreich operieren. Der Hohepunkt dieser Entwicklung ist der Versuch, die Atome selbst aus Elektronen aufzubanen; da in dieser Zeitschrift in letzter Zeit mehrfach über diese Arbeiten, die sich an die Quantenhypothese Plancks anschließen, berichtet worden ist, wollen wir hier nicht darauf eingehen ${ }^{*}$ ).

Dagegen wollen wir über einige Fortschritte berichten, die den oben geschilderten Weg rom mechanischen zum elektrischen Weltbilde der Physik besonders grell beleuchten.

Sie betreffen die Konstitution der festen Körper und die Natur der Kräfte, die sie zusammenhalten. Die festen Körper sind entweder amorph (glasig) oder kristallinisch. Doch pflegt man heute nur die Kristalle (bzw. Gemenge von

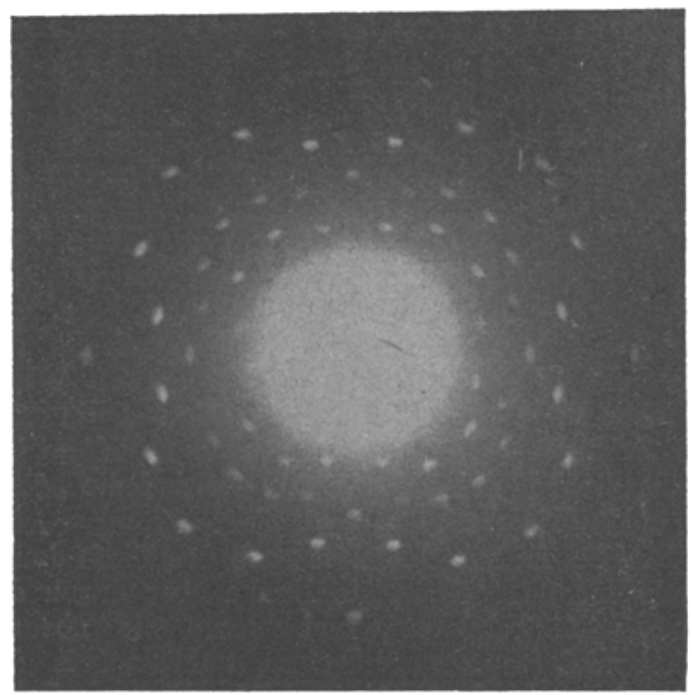

Fig. 1.

Kristallen, wie dio meisten Metalle) im eigentlichen Sinne als fest anzusprechen, während die amorphen Substanzen in vieler Hinsicht als besonders zähe Flüssigkeiten zu gelten haben.

Das Wesen der. Kristalle ist ihr regelmäBiger Aufbau aus den Atomen und Molekeln. Dieser kann, wie v. Laue entdeckt hat, mit Hilfe von Röntgenstrahlen direkt auf der photographischen Platte sichtbar gemacht werden ${ }^{2}$. Fig. 1 gibt

1) Ausfubrliche Darstellungen der Quantentheorie und ihrer Anwendungen auf die Atomstruktur findet man in dem Planck-Hefte dieser Zeitschrift (6. Jahrg. Heft 17, 1918).

2) Darstellungen dieser Entdeckung und ihrer Ausarbeitung sind in dieser Zeitschrift mehrere erschienen: $H_{n}$ Löwy, 1. Jahrg., S. 105, 1913. A. Sommerfeld, eine solche Aufnahme eines Steinsalzkristalls (NaCl) wieder'). Wir kennen heute die Feinstruktur oder das "Gitter" einer großen Anzahl von Kristallen; so ist z. B. das Steinsalz aus $\mathrm{Na-}$ trium- und Chloratomen nach Art eines, dreidimensionalen Schachbretts" aufgebaut, wie die Fig. 2 zeigt. Die Kristalle sind also in Wirklichkeit gar keine kontinuierlichen Medien. Es ist vielmehr eine Aufgabe, deren Lösung viel Mühe und Scharfinn gekostet hat, nachzuweisen, daß solche Raumgitter bei grober Betrachtung sich mechanisch wie die kontinuierlichen Festkörpe: der alten Elastizitätstheorie verhalteu. Dex Ausgangspunkt der mechanischen Äthertheorien ist damit eigentlich von Grund aus zerstört; denn diese gehen, von dem kontinuierlichen Festkörper als einer durch ursprüngliche Erfahrung oder Anschaung gegebenen Grundlage aus und fordern die Zurückführung aller andern Phänomene auf die Eigenschaften solcher. Medien. Nun ist aber doch die kontinuierliche, elastische Substanz" einfach eine Täuschung, hervorgerufen durch die Grobheit unserer Sinne. Die scheinbaren Kontinua sind in Wirklichkeit diskontinuierliche Git. ter, zwischen ihren Atomen sind Lücken. Denkt man diese durch A ther erfiallt, welchen Sinn hat es dann noch, ihm Eigenschaften zuzuschreiben. die dem Verhalten der Gitter im groben, als scheinbare Kontinua, extsprechen? Dies hieBe offenbar, sich im Kreise drehen.

Die heutige Physik nimmt darum einen andera Standpunkt ein, der nicht nur konsequenter ist, sondern auch zu greifbaren Resultaten geführt hat. Das elektromagnetische Feld im Äthen wird als empirisch gegeben hingenommen. Der feste Körper, der Kristall, ist eine regelmäBige Anordnung elektrischer Ladungen, die nach den Feldgesetzen aufeinander wirken. Hieraus entspringen jene groben Eigenschatten, die das Kristallgitter mit dem elastischen Kontinum der alten Mechanik gemein hat.

Diese Auffassung führt nun zu dem SchluB: die mechanischen, elastischen Kräfte der fester Körper sind in Wahrheit-elektrische Kräfte. Wenn wir die Ladungen und Abstände der Atome in den Kristallgittern kennen, sind aber alle elektrischen Wechselwirkungen grundsätzlich bekannt; es muB also möglich sein, die elastischen Eigenschaften quantitativ vorauszuberechnen.

An dieser Stelle gelangt man uber die Streitfragen der theoretischen Auffassung hinaus $z u$ Problemen, deren Lösung die Überlegenheit des neven Standpunkts durch zahlenmäBige Rechnumg erweisen wärde $)$. Der Weg zu diesey" Lösung

4. Jahrg.: S. 1, S. 13, 1916. F. Rinne, 5. Jahrg., \$. 48 1917. M. Siegbahn, 5. Jahrg., S. 512, S. 528, 1917: außerdem zahlreiche kleinere Mitteilungen.

1) Die Figur stammt aus F. Rinne, Beiträge zur Kenntnia der Kristall-Röntrenogramme 1. Mittejlung. Ber. d. math.-phys. Kl, d. kgl. sächs. Ges, d. Wiss, zu Leipzig. LXVIT. Bd., S. 303, 1915.

2) In der kinetischen Gastheorie verhielt es sich Mhnlich. Auch hier bestand der Gegensatz zwischen 
war ziemlich weit. Erst muBten die Zusammenhänge aller möglichen mechanischen, thermischen, elektrischen, optischen Eigenschaften der Kristalle auf Grund der Gistertheorie geklärt und formal auf die Kräfte zwischen den einzelnen Partikeln des Kristallgitters zurückgeführt werden'). Wir wollen die wichtigsten Ergebnisse dieser Untersuchungen an dem Beispiel des oben erwähnten Steinsalzkristalls erläutern.

Wie wir schon sahen, ist das Gitter dieses Kristalls nicht aus NaCl-Molekeln, sondern aus einzelnen Nartomen und Cl-Atomen aufgebaut (Fig. 2); dies wird nicht nur durch die Röntgenaufnahmen nach $v$. Laue bewiesen, sondern auch durch das thermische Verhalten des Kristalles. Die Wärme der Körper besteht in Bewegungen ihrer kleinsten Teile; bei den Kristallen sind es Sohwingungen um Gleichgewichtslagen. Nach einem fundamentalen Satz der statistischen Mechanik verteilt sich bei höheren Temperaturen die Energie dieser Schwingungen auf die einzelnen

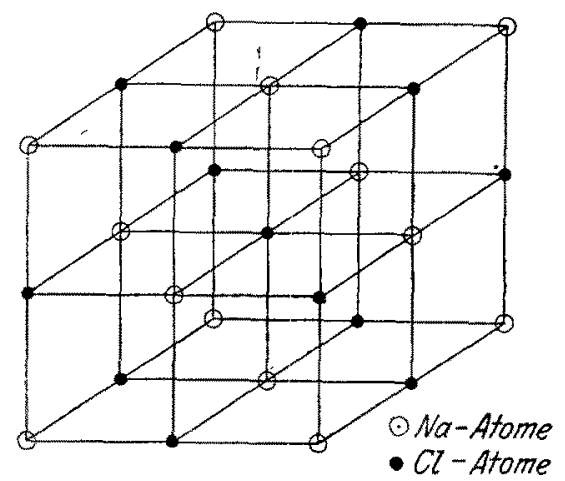

Fig. 2.

Partikel so, daß im Wittel über längere Zeit jedes Partikel dieselbe Fnergie bekommt, deren Betrag aus der kinetischen Gastheorie wohl bekannt ist, gleichgültig, wie das Partikel beschaffen ist. Mit Hilfe von thermischen Messungen läßt sich somit feststellen, wieviele Partikel, jedes mit diesem Energiebetrage versehen, in der Masseneinheit vorhanden sein müssen, damit dex beobachtete Wämeinhalt herauskommt. Man findet so, dab die Partikel nicht die $\mathrm{NaCl}$-Molekel sein können; denn dann hätte die Masseneinheit nur halb so viele Partikel, als im Falle selbständiger Atome, also auch nur die Hälfte des beobachteten Wärmeinhalts.

Löst man ein Stück Steinsalz in Wasser auf, so wird die Lösung ein Leiter des elektrischen

Kontinuums- und Atomtbeorie, die so lange gleichwertig blieben, als die letztere nur die formalen $\mathrm{Zu}$ sammenhänge darstellen konnte; erst als os der atomistischen Auffassung gelang, Koeffizienten der For meln zahlenmäBig zu bestimmen (wie das Verhältnis der spezifischen. Wärmen, das Verhältnis der Koeffizienten von Wärmeleitung und innerer Reibung wow.), war ihr Sieg gesichert.

1) M. Born, Dynamik der Kristallgitter (B. G. Teubner, Leipzig, 1915).
Stroms, ein sogenannter Elektrolyt. Ein solcher unterscheidet sich von einem metallischen Leiter dadurah, daß der Stromdurchgang mit Transport der gelösten Materie verknüpft ist; das zeigt sich daran, daß sich an den Eintritts- und Austrittsstellen des Stroms (den Elektroden) Natrium bzw. Chlor abscheidet. Faraday hat das Gesetz dieses Vorgangs entdeckt; er fand, daß beim Durchgang einer bestimmten Elektrizitätsmenge immer die gleichen Mengen der beiden Stoffe abgeschieden werden, und zwar solche Mengen, die sich chemisch gerade zu der Verbindung $\mathrm{NaCl}$ absättigen (,äquivalent" sind). Man deutet das atomistisch durch die Annahme, daß jedes Atom die gleiche Elektrizitätsmenge transportiert; das Na-Atom trägt ein positives, das Cl-Atom ein negatives Elektrizitätsatom, Man nennt solche geladene Atome "Ionen". Dieses Elektrizitütsatom hat man bei vielen andern Erscheinungen wieder gefunden, allerdings immer nux das negative; man hat es „Elektron“ genannt. Die positive Ladung ist immer an die Materie gebunden. Das neutrale Atom besteht aus einem positiven Kern, der von einer Anzahl von Elektronen umgeben ist. Das Cl-Ion ist gewissermaben die chemische Verbindung eines neutralen Cl-Atoms mit einem Elektron. Das positive Na-Ion muß man sich dadureh entstanden denken, daß dem Bestande des neutralen $\mathrm{Na}$-Atoms ein Elektron entrissen ist. Die GröBe der Ladung des einzelnen Elektrons ist eine bestimmte Naturkonstante, die man auf manuig. faltige Weise bestimmen kann; sie beträgt $e=$ 4,76 $\cdot 10^{-10}$ elektrostatische Einheiten.

Wenn man eine solche NaCl-Lösung eindampft, so daf das Steinsalz auskristallisiert, so besteht der Vorgang darin, daß sich die einzelnen $\mathrm{Na}$ - und Cl-Atome zu dem oben besprochenen Gitter zusammensetzen. Was wird dabei aus der Ladung der Atome? Tauschen sie diese aus oder behalten sie auch im festen Zustande ihren Ionencharakter?

Die Antwort auf diese Frage hat Madelung ${ }^{1}$ ) gegeben durch die Deutung einer optischen Eigenschaft der Kristalle wie NaCl. Denken wir uns etwa ein Stück Steinsalz zwischen zwei parallele, geladene Metallplatten gebracht; dann ist das in Fig. 2 dargestellte Gitter einem elektrischen Folde ausgesetzt (Fig. 3). Da nun die $\mathrm{Na}$-Atome positiv, die Cl-Atome negativ geladen sind, werden die ersteren in der Richtung des Feldes, die letzteren in entgegengesetzter Pichtung eine Kraft exfahren; diese wird wegen der Festigkeit des Kristallgefüges im allgemeinen nur äuBerst kleine Verschiebungen der Na- gegen die Cl-Ionen hervorrufen. Man erinnere sich nun aber an die bekannte Erscheinung der Resonanz; wenn man eine Schaukel im Rhythmus ihrer eignen freien Schwingungen anstöBt, kann man sie mit äuBerst geringer Anstrengung zu großen Ausschlägen bringen, Ebenso wird ein periodisch wechselndes elek-

I) E. Madelung, Nachr. d. K. Ges. d. Wiss. zu Göttingen, math.-phys. Kl. 1909, 1910. 
trisohes Feld, wenn sein Rhythmus geeignet gewählt wird, das Kristallgefüge äuBerst heftig ersehüttern kơnnen. Die Lichtwellen sind solche periodischen elektrischen Felder; man wird also erwarten dürfen, daB Licht von geeigneter Schwingungszahl den Kristall merklich beeinfluBt nnd natürlich umgekehrt von ihm beeinflußt wird. Rubens ${ }^{1}$ ) hat diese Wirkung tatsächlich entdeckt; ef fand, dab sehr langwelliges (nicht mehr sichtbares) Licht von bestimmter Schwingungsdauer von dem Kristall äußerst stark reflektiert wird, wherend Licht von ein wenig anderer Schwingungsdauer unbeeinflußt hindurchgeht. Durch mehrfache Reflexionen konnte er dieses selektiv reflektierte Licht sauber isolieren und nanute es deswegen „Reststrahlen".

Die Reststrahlen beweisen die Ladung der Ionen; das diese bei Kristallen vom Typus $\mathrm{NaOl}$ genau ein Elektron beträgt, hat man dadurch zeigen können, dab die Sohwingungsdauer sich aus der Elektronenladung, den Massen der Ionen und den meBbaren elastischen Kräften des Kristalls richtig berechnen läbt.

Neverdings ist die Ionenladung von Debye uad Scherrer ${ }^{2}$ ) aber auch direkt durch Röntgen-

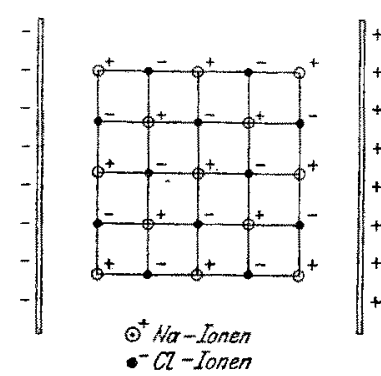

Fig. 3.

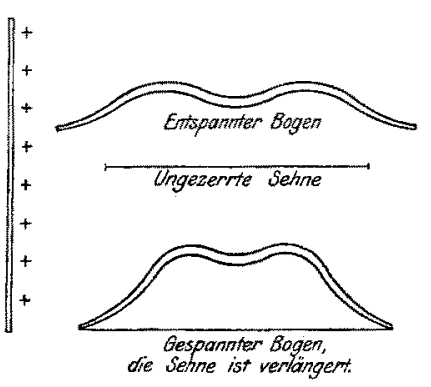

Fig. 4. aufnahmen nachgewiesen worden. Die Röntgenwellen erschüttern die Elektronen des Atoms und erzeugen dadurch sekundäre Wellen, die von den Atomen ausgehen; diese liefern durch ihre Interferenz die von Laue entdeckten Erscheinungen (Fig. 1). Die Stärke der Interferenzflecke wird somit von der Anzahl der in den Atomen vorhandenen Elektronen abhängen; umgekehrt kann man durch Ausmessen der Stärke der Interferenzflecke oinen Rückschluß auf die Anzahl der Elektronen der Atome ziehen. Auf diese Weise wurde für den Kristall Lithiumfluorid gefunden, daB das LiAtom ein Elektron zu wenig, das F-Atom eines zu viel hat gegenüber dem neutralen Zustande. Das Fntsprechende gilt unzweifelhaft für alle Salze der Alkalimetalle (Li, $\mathrm{Na}, \mathrm{K}, \mathrm{Rb}, \mathrm{Os}$ ) mit den Halogenen (F, Cl, $\mathrm{Br}, \mathrm{J}$ ).

Damit haben wir die Tatsachen gesammelt, die uber die Konstitution der Kristalle bekannt

1) Nichols u. Rubens, Wied. Ann. 60, 438, 1897 Rubens i. Aschkinass, Wied. Ann. 67, 459, $1899 . \quad H$. Rubens, Wied. Ann. 69, 576, 1899, und viele weitere Arbeiten. 1918.

2) P.Debye u. P. Sokerrer, Phys. Zeitschr. 19, \$. 474 , sein müssen, ehe an eine absolute Berechnung ihrer mechanischen Eigenschaften aus den elektrischen Daten gegangen werden $\operatorname{kann}^{\mathbf{1}}$ ).

Stellen wir uns nun den Steinsalzkristall mit seinen abwechselnd geladenen $\mathrm{Na}$ - und Cl-Atomen vor (Fig. 2), so sehen wir sofort, dal aus den Ladungen notwendig ein Kontraktionsbestreben des Gitters folgt; denn benachbarte entgegengesetzt geladene Teilchen zichen sich an, gleiche Teilchen stoBen sich zwar ab, aber wesentlich schwächer, weil sie weiter voneinander abstehen und die elektrische Kraft nach dem bekannten Gesetz von Coulomb umgekehrt wie das Quadrat der Entfernung abnimmt.

Die strenge Berechnung dieses Kontraktionsbestrebens ist neuerdings Madelung ${ }^{2}$ ) gelungen. Er hat folgendes gezeigt: Man denke sich zunächst alle Tonen in unendlicher Entfernung voneinander und baue sie dann unendlich langsam zu dem Kristallgitter zusammen; wegen des Kontralstionsbestrebens leisten sie dabei eine gewisse Arbeit, die von der absoluten Dimension des Gitters abhängt. Ist $\delta$ der Abstand zweier längs einer Wurfelkante benachbarter gleicher Tonen eines Kristalls vom Typus $\mathrm{NaCl}$, so findet man für diese Arbeit 13,94 $e^{2} / \delta$, wo $e$ die oben genannte Ladung des Elektrons ist.

Warum bleiben nun aber die Ionen in diesem bestimmten, durch 8 gemessenen Abstande stehen? Warum stürzen sie nicht ganz zusammen? Das läßt sich offenbar nur durch eine Abstoßungskraft erklären, die bei groBer Annäherung wirksam wird und dem Kontraktionsbestreben das Gleichgewicht hält. Überlegungen sehr allgemeiner Art führen darauf, für die Arbeit, die diese Kraft bei dem soeben geschilderten Prozeß dex Entstehung des Kristalls aus isolierten Ionen leistet, einen Ausdruck der. Form b/sn anzusetzen, wo $n$ eine ganze $Z$ ahl und $b$ eine Konstante ist; wie nämlich das strenge Kraftgesetz auch beschaffen sein mag, wenn es auf dem Zusammenwirken der elektrischen Ladungen des Atominnern beruht, muß es sich doch mitr großer Näherung durch eine solche Formel darstellen lassen.

Die Konstante $b$ aber läßt sich durch den Ionemabstand ausdrücken. $\mathrm{Lm}$ das einzusehen, betrachte man als Modell fur das Gleichgewicht einer kontrahierenden and einer dilatierenden Kraft (Fig, 4) einen Bogen, jene Waffe primitiver Völker. Das Holz strobt sich gerade zu strecken, die Sehne zieht die Enden zusammen. Wïrde man die Kraft der elastischen Biegung des Holzes und der astischen Dehnung der Sehne kennen, so würde sich daraus die tatsächliche Gleichgewichtsfigur des Bogens und die Länge der Sehne (die etwa dem Tonenabstand 8 analog ist) berechnen lassen. Denkt man sich nun nur die Kontraktionskraft der Sehne, aber nicht die Dilatafionskraft des Holzbogens bekannt, aber:

1) M. Barn u. A. Landé, Verh. d. Deutsch. Phys. Ges. $20,187,1918$.

2) D. Ifadelung, Phys. Zeitschr. 19, S. 524, 1918. 
außerdem die Sehnenlänge bestimmt, so kann man umgekehrt die Kraft des Holzbogens aus den beiden gegebenen. Größen berechnen. Das entspricht nun genau unserem Falle: die Kontraktionskraft ist bekannt, der Ionenabstand $\delta$ läBt sich aus der Dichte und den Atomgewichten einfach berechnen, folglich liefert die Gleichgewichtsbedingung eine Beziehung zur Berechnung der Abstobung $6 / 8 x$. Unbekannt bleibt dann nur noch der Exponent $n$.

Bis auf diesen ist also die bei Entstehung des Kristalls aus seinen Ionen geleistete Arbeit vollständig bekannt, und damit sind nach allgemeinen Gesetzen der Dynamik alle von dem Ionenabstand $\delta$ abhängigen Eigenschaften des Kristalls mitbestimmt. Setzt man den Kristall einem gleichförmigen, allseitigen Drucke aus, so wird er komprimiert; diese Volumenänderung ${ }^{2}$ )

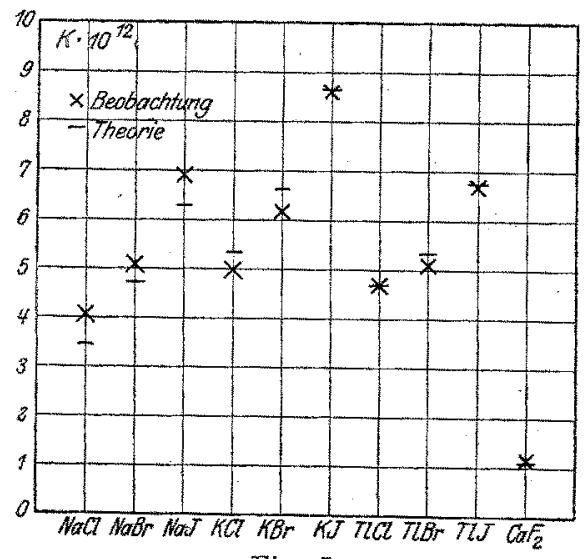

Fig. 5.

hängt offenbar nur von der Anderung des Ionenabstands $\delta$ ab, muß sich also aus unseren Prinzipien ableiten lassen. Das Verhältnis der relativen Volumenänderung zu der dazu aötigen Druckzunahme heiBt Kompressibilität und wird mit $x$ bezeichnet. Es muß also möglich sein, bei geeigneter Wahl des ganzzahligen Exponenten $n$ dieses * allein aus der - Ladung des Elektrons $e$ und dem Ionenabstand $\delta$ (der wieder dureh Dichte und Atomgewichte gegeben ist) zu berechnen. Mit $n=9$ hat sich eine sehr gute Ubereinstimmung ergeben, die durch Fig. 5 veranschaulicht wird ${ }^{2}$ ). Darin sind die von Richards und Jones ${ }^{3}$ ) gemessenen Kompressibilitäten in der Einheit 10-12 dyn/cm als Kreuze, die nach unserer Theorie berechneten Werte als Striche eingetragen. Auber 9 Salzen vom Typus $\mathrm{NaCl}$ enthäl die Figur auch noch den Kristall FluBspat $\mathrm{OaF}_{2}$, bei dem ebenso gute Ubereinstimmung herrscht; das ist besonders

1) Bei dem Beispiel unseres Bogens entspricht diese Volumenänderung etwa der Annäherung der Sehnenenden, wenn der Bogen (zum Schusse) graspannt wird. Bei gegebener Spannkraft ist diese Annüherung offenbar dureh die elastischen Eigenschaften von Bogen und Sehne völlig bestimmt.

2) Mi. Born und A. Landé, I. c.

3) Th. W. Richard's und Gr. Jones, Journ. Amer. Chem. Soc. 31, 158, 1909. beweisend für die Richtigkeit der Theorie, weil dieser Kristall ein ganz anderes Gitter und daher das Kontraktionsbestreben einen ganz andern Zahlenwert hat $\left(38,7 e^{2 / \delta}\right)$.

Damit ist der Nachweis erbracht, daß der elastische Widerstand gegen Zusammendrücken anf elektrischen Kräften beruht und im voraus berechnet werden kann. Man wird wohl nicht zögern, dieses Ergebnis zu verallgemeinern.

Es wäre nun noch viel zu sagen über die Bem deutung des Exponenten $n=9$; dieser Zahlenwert wirft helles Licht auf den inneren Bau der Tonen, die mit großer. Wahrscheinlichkeit keine Systeme von ebenen Elektronenringen (nach Bohr) sind, sondern die Form von Würfeln haben. Die Zahl 8 der Würfelecken wieder steht wahrscheinlieh in engem Zusammenhang mit dem periodischen System der Elemente, dessen ersten beiden Perioden die Länge 8 haben. Auch zeigen sich Beziehungen zwischen chemischen Wärmetönungen und den Ionisierungsspannungen ${ }^{1}$ ) und manche andere Ausblicke. Doch sind diese Dinge noch zu sehr im Stadium der Entwicklung, um an diesem Orte dargestellt zu werden. Wir können unsere Ausführungen mit der GewiBheit schlieben, daB die Wissenschaft ein Stück des Weges rom ,mechanischen Ather" zux ,elektrischen Materie" glücklich überwunden hat.

\section{Die Reste fossiler Tiere im Volks- glauben und in der Sage.} Von Dr. Othenio Abel,

a. o. Professor der Paläobiologie an der Wiener Universität. (SehluB.)

Wie durch die Tntersuchungen Schraders (Sitzungsber. d. Berliner Akad. d. Wiss., 1892) wohl endgültig aufgeklärt wurde, geht die Einhornsage auf die Darstellungen des Ur oder Auerochsen (Bos primigenius) zurüak, die sich auf altassyrisch-babylonischen Reliefs vorfinden. Diese Darstellungen sind ebenso wie die ägyptisehen Wandmalereien and Reliefs streng im Profil wiedergegeben, so daB nur ein. Horn abgebildet erscheint. Die Perser ahmten diese Abbildungen nach, ohne das Tier selbst zu kennen, da zur Zeit des Baues des Königspalastes von Persepolis der $\mathrm{Ur}$ in Mesopotamien bereits ausgestorben war. Der griechische Arzt Ktesias, Leibarzt von Artaxerxes IT., sah diese Darstellungen in Persepolis und brachte von hier die Nachricht ron dem merkwürdigen Einhorn nach Hause. Aristoteles, Plinius und Aelianus haben diese Schilderungen übermommen, doch scheint in dieser und in späterer Zeit die Vorstellung des Einhorns durch die auf ägyptischen Reliefs dargestellten Säbelantilopen (Oryx) beeinflußt worden zu sein, die in stren-

1) Die Ionisierungsspannung ist diejenige Spannung, die nötig ist, um einem Atom ein Elektron au entreißen; sie ist nach den Untersuchungen von Franck ind Herte (Verh. d. Deatseh. Phys, Ges, 15, 34, 1913) eine charakteristigche Konstante des Atoms. 